\title{
5
}

\section{Interoperability of Distributed Platforms: a Compatibility Perspective}

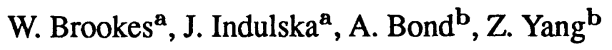

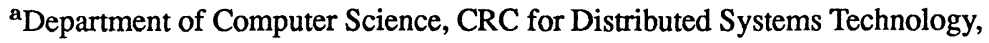 \\ The University of Queensland, Brisbane 4072, Australia \\ ${ }^{\text {b } C R C ~ f o r ~ D i s t r i b u t e d ~ S y s t e m s ~ T e c h n o l o g y, ~ L e v e l ~ 7, ~ G e h r m a n n ~ L a b o r a t o r i e s, ~}$ \\ The University of Queensland, Brisbane 4072, Australia
}

Interoperability of heterogeneous distributed platforms is affected by differences in their type systems, including both differences in syntax and semantics of types and approaches to inclusion polymorphism. This paper examines the differences of well known distributed platforms (DCE, ANSAware, CORBA) as well as models for distributed and object oriented systems. The examination is illustrated by a scenario of their cooperation. The scenario is based on resolving differences during the federation process. The federation involves extension of persistent type repositories for each domain to incorporate mappings among domains. The scenario focuses on differences in compatibility relationships.

Keyword Codes: C.2.4; D.2.6

Keywords: Distributed Systems, Programming Environments

\section{INTRODUCTION}

Current trends in distributed computing are towards creating open distributed systems, where co-operation is necessary in spite of the heterogeneity and autonomy of systems present in an open environment. The open, heterogeneous nature of the environment introduces a number of difficult problems which are not encountered in centralised systems, or in closed homogeneous systems. The open nature of the system means that objects are created and destroyed frequently, and more importantly, new types of objects are frequently created and objects with new types are required to interwork with existing components. Late binding is assumed as a mechanism to allow existing objects to work with newly introduced components.

A number of different distributed computing platforms attempt to address these issues (to different extents) including DCE [21, 22], CORBA (and OMA) [17, 18], ANSAware [2] and

The work reported in this paper has been funded in part by the Cooperative Research Centres Program through the Department of the Prime Minister and Cabinet of the Commonwealth Government of Australia. It was also partially supported by an Australian Government Postgraduate Research Scholarship (APRA). 
COMANDOS [7]. Also, the standard under development, the ISO Reference Model of Open Distributed Processing (RM-ODP) [12], attempts to create a framework for interoperability of open distributed systems. Moreover, research on type-safe interoperability of object-oriented applications $[1,13,14]$ adds additional insight into the problem.

This paper examines type-safety issues related to interoperability of heterogeneous and autonomous platforms. We analyse the requirements for type-safe interoperability and examine some existing platforms for distributed computing against these requirements showing the differences in type models which make interoperability difficult. The examination is supported by both a scenario of interoperability for these platforms and a proposal for possible solutions. The scenario focuses on differences in compatibility relationships defined for the platforms.

For the analysis, we assume an object-based model of distributed systems, similar to that described in RM-ODP. Using this model, an open distributed system consists of a large number of heterogeneous communicating objects managed by many independent, autonomous authorities. Meaningful interoperability requires that objects can be discovered, bound to, dynamically invoked and also replaced by compatible objects at run time. Therefore, if the objects are from different type models, either a common framework which will allow mapping between the objects is required [15], or one-to-one mappings have to be provided. The framework or oneto-one mappings must capture basic differences in the syntax of types, but also some semantic differences including differences in compatibility relationships.

A large amount of research has been already carried out on a unifying type model supporting interoperability $[5,6,7,9,12,15,16,19,20]$. As this work approaches the problem from different backgrounds, a unification of the models is still necessary. In the meantime, cooperation between distributed platforms can be achieved by a one-to-one mapping approach. In this paper we analyse such a mapping between type systems of distributed domains. The goal of the analysis is twofold: to show the extent of interworking between platforms if one-to-one mapping is assumed and to facilitate work on a common framework by highlighting the differences which should be captured in the common model.

The remainder of the paper is organised as follows. Section 2 discusses requirements for type-safe interoperability of distributed systems. Section 3 presents approaches of existing platforms considering the mapping of types and relationships. Section 4 illustrates the problems involved using a case study of existing distributed system models and showing an approach to mapping relationships between these models.

\section{REQUIREMENTS FOR DISTRIBUTED PLATFORM INTEROPERABILITY}

Interoperability requires that resource type selection is possible during resource discovery and the same for type matching during binding and invocation. Both selection and matching can cross platform boundaries (therefore also type system boundaries) and should also work for evolving systems. In spite of crossing boundaries between different type systems, the operations (resource selection, binding and invocation) should be type safe, i.e. free from mismatched types. Many object-oriented languages and platforms for distributed computing already provide descriptions of objects, their interfaces and also a definition of compatibility which differs for different platforms [19]. Therefore, the features of the type models of existing languages which are visible during interoperability, i.e. interaction types (operations, streams, notifications, etc.), interface types, binding types and relationship types have to be captured in a common framework 
which will allow mapping between models.

Supporting such mapping implies that the framework must include a means for the description of some elements of objects' semantics and some semantics of relationships. When type matching is performed in an open system to search for compatible types, as much as possible of this matching should be performed automatically and invisibly to users of the system. In the general case, it is not always possible to perform transparent, automatic type matching when type semantics are involved, but the type model should be designed with support for all kinds of matching: automatic, semi-automatic or manual. Even in the latter case, when matching is performed manually by a user it should be supported by a description of elements of semantics which will facilitate the user's task of determining matches.

In addition to substitutability, the framework should allow the creation of generic objects. This implies that the model should capture two kinds of polymorphism which support genericity and substitutability, i.e.

- parametric polymorphism allowing definition of generic operations/objects,

- inclusion polymorphism defining substitutability (subtyping/compatibility) rules, especially substitutability of interface types and relationship types which may involve semantics.

To create such a framework, the model should be able to describe both the structure and some semantics of data types, action types (interaction types, interfaces, bindings between objects) and relationship types. Existing Interface Definition Languages [2, 3, 18, 21, 22] and their type models aim to provide a description of types visible during interoperability. They support polymorphism to a certain extent, however the models do not address all the requirements and there is still a need for a richer and unifying framework. The type model encapsulating these features visible at platform boundaries is necessary if interworking of heterogeneous platforms is to be supported.

In addition the model should be extensible to encompass new domains and their type models, and therefore the ability to dynamically learn about new object types or new relationship types is necessary.

In the next section, we compare the syntax and semantics of types of data, actions and relationships in type models of existing distributed and object-oriented platforms. Type safety of their type matching operations is also considered.

\section{EXISTING APPROACHES}

Existing platforms for distributed computing have adopted their own type models to support application interoperability. The approaches that are compared here include DCE [21, 22], OMG's CORBA (and OMA) [17, 18], ANSAware [2], RM-ODP-based systems [12] and work on type-safe interoperability of object-oriented applications including Liskov \& Wing's work $[13,14]$. and the POOL language [1].

All of these models provide features for supporting interoperability to different degrees. The three distributed environments (DCE, CORBA and ANSAware) and the POOL language all provide a standard set of basic data types and constructors. DCE has a richer set than the other systems, but all provide a set which is satisfactory for supporting application interoperability. 
However, neither set is 'canonical' in the sense of being more general than other models. RM-ODP does not specify data types important for interoperability.

In terms of action types, all models at least support the concept of operations (methods). All models also support either the concept of interfaces (logical groupings of operations) or objects or both. DCE, CORBA, ANSAware and RM-ODP also support notifications, and RMODP supports signals (one way typed messages). Of the five models, only America (POOL language) and Liskov \& Wing provide any description of semantics of action types, where the semantics are those of the action itself, not the semantics of the language in which the actions are described. Liskov \& Wing use invariants and pre- and post-conditions to describe the semantics of operations. The POOL language uses properties to capture semantics of operations and objects, however the designers note the need for more expressive semantic description [1].

In terms of supporting relationships between types, only the OMG approach supports the notion of multiple, user-defined relationships, through the use of the Relationships Service. The other models use only built-in relationships (usually for inclusion polymorphism, namely subtyping or inheritance).

DCE supports interface subtyping through the use of versions. If one interface has the same major version and a larger minor version number, then it is a subtype. Subtyping is not part of the DCE IDL language, and is only an abstract concept defined on operation signatures. DCE uses interface subtyping by extension, namely that one interface is a subtype of another if the subtype has at least the same operations as the supertype, and possibly more. Additionally, the operations of the subtype must be in the same order as the supertype. Arguments and results of the operations must be of exactly the same type. It does not include any semantics of the subtyping relationship.

In addition to the user-defined relationships in the Relationships Service, CORBA defines interface inheritance based on type syntax (not semantics) supporting interface extension by the addition of operations. OMA, the OMG architecture [17] at a higher level than CORBA, recognises the need for a subtyping relationship. OMA also notes that subtyping and inheritance are different concepts, although inheritance often implies subtyping. OMA defines subtyping as follows:

Formally, if $S$ is declared to be a subtype of $T$ (and conversely, $T$ is a supertype of $S$ ), then for each operation $o_{i} \in O p s(T)$ there exists a corresponding operation $o_{j} \in O p s(S)$ such that the following conditions hold:

1. the name of the operations match

2. the number and types of the parameters are the same

3. the number and types of the results are the same.

Thus, for every operation in $T$ there must be a corresponding operation in $S$, though there may be more operations in $O p s(S)$ than $O p s(T)$.

ANSAware supports interface inheritance through an is_compatible_with relationship. This information is not used in ANSAware, apart from for reuse. ANSAware also defines a subtyping relationship in the Type Manager (type conformance) which it assumes is related to the interface inheritance. The ANSAware Type Manager stores only names in the subtyping graph (based on information supplied by a user), and type descriptions are not taken into account when determining subtyping. Again the relationship is only based on syntax. It is very similar to 
subtyping in DCE in that it only supports subtyping by extension, assumes that argument and result types are identical and requires the same order of operations.

RM-ODP also defines interface subtyping. Here we will only consider RM-ODP's operational interfaces. The difference between the RM-ODP subtyping definition and that of DCE, OMG and ANSAware is the addition of subtyping of operation arguments and results, in addition to subtyping by extension (allowing extra operations). Parameter subtyping follows the "contravariant arguments, covariant results" rule [19]. RM-ODP [12] informally defines its subtyping rules as follows (a more rigorous definition of the subtyping rules are also included in RM-ODP):

Operational interface $X$ is a subtype of interface $Y$ if the conditions below are met:

- for every operation signature in $Y$, there is an operation signature in $X$ (the corresponding signature in $X$ ) which defines an operation with the same name;

- for each signature in $Y$, the corresponding signature in $X$ has the same number and names of arguments;

- for each signature in $Y$, every argument type is a subtype of the corresponding argument type in the corresponding signature in $X$;

- the set of termination names of an operation signature in $Y$ contains the set of termination names of the corresponding signature in $X$;

- for each operation signature in $Y$ a given termination in the corresponding signature in $X$ has the same number and names of results in the termination of the same name in the signature in $Y$;

- for each operation signature in $Y$, every result type associated with a given termination in the corresponding signature in $X$ is a subtype of the result type with the same name in the same termination name, in the signature in $Y$.

Liskov \& Wing's major contribution is their definition of an interface subtyping relationship which includes subtyping of operations based on described semantics as well as syntax. In addition, it allows for the renaming of operations by a subtype. Semantics are described as preand post-conditions in a language based around the Larch specification languages [10], and the subtyping is based on the weaker precondition and stronger postcondition rule [13, 14]. It also includes syntax-based subtyping based on contravariant arguments and covariant results. POOL is a language which incorporates the theory of behavioural subtyping, but in the implementation restricts the behaviour description to the use of properties to tag behaviour [1].

Both approaches to behavioural subtyping describe operation behaviour using pre- and postconditions specified as state predicates. To formally relate a subtype to a supertype, Liskov \& Wing define a correspondence mapping consisting of an abstraction function for relating values of the subtype to values of the supertype, a renaming function to relate subtype operations to supertype operations and an extension map to explain the effects of any additional operations provided by the subtype. If such a correspondence mapping can be found between two types, subject to both structural and semantic subtyping rules, then one type is a behavioural subtype of another. Using an informal notation, the semantic subtyping defined by Liskov \& Wing is given by the following two rules:

1. pre (super) $\Rightarrow$ pre (sub)

2. (pre (sub) $\Rightarrow$ post (sub) ) $\Rightarrow$

( pre (super) $\Rightarrow$ post (super) ) 
where pre (super) is the precondition of the supertype operation, post (super) is the postcondition of the supertype operation, pre (sub) is the precondition of the subtype operation and post (sub) is the postcondition of the subtype operation. The first rule known by Liskov $\&$ Wing as the pre-condition rule and the second as the predicate rule. POOL uses a slightly different (and stronger) formulation, but is very similar in approach.

POOL is the only model that supports parametric polymorphism, through its use of 'bounded genericity'. CORBA supports universal polymorphism through introducing the type ANY, however this is a weaker form of polymorphism than true parametric polymorphism where it is possible to create type templates which can be parameterised to create actual types [8].

As discussed earlier, there are three basic points at which type matching can occur in a distributed environment: type selection, binding and invocation of interactions. DCE supports matching (based on versions) during selection and binding only, and not during invocation. CORBA assumes matching at all three points. ANSAware supports matching during selection, but not during binding or invocation. RM-ODP assumes matching is supported at all three points. Both Liskov \& Wing's approach and the POOL language are models for object-oriented languages, and thus it is irrelevant to consider when matching occurs (as models do not address concrete issues such as selection, binding and invocation).

Of all the approaches listed, they can be clearly divided according to the definition of the subtyping relationship they use. DCE, ANSAware and CORBA all only provide subtyping based on extension, that is, the addition of operations to a subtype. RM-ODP takes this one step further and also supports subtyping of operation arguments and results. Both Liskov \& Wing's approach and the POOL language support behaviour description, fully in theory, and limited in practical implementation. In addition, Liskov \& Wing's subtyping definition supports renaming.

The differences in subtyping relationships are summarised in Table 1.

\begin{tabular}{|c||c|c|c|c||c|c|c|}
\hline \multicolumn{1}{|c||}{} & \multicolumn{3}{c||}{ subtyping definition } & \multicolumn{3}{c|}{ type safety } \\
\hline & $\begin{array}{c}\text { interface } \\
\text { extension }\end{array}$ & $\begin{array}{c}\text { parameter } \\
\text { subtyping }\end{array}$ & $\begin{array}{c}\text { behaviour } \\
\text { description }\end{array}$ & $\begin{array}{c}\text { component } \\
\text { renaming }\end{array}$ & selection & binding & invocation \\
\hline \hline DCE & $\checkmark$ & & & & $\checkmark$ & $\checkmark$ & \\
\hline OMA/CORBA & $\checkmark$ & & & & $\checkmark$ & $\checkmark$ & $\checkmark$ \\
\hline ANSAware & $\checkmark$ & & & & $\checkmark$ & & \\
\hline RM-ODP & $\checkmark$ & $\checkmark$ & & & $\checkmark$ & $\checkmark$ & $\checkmark$ \\
\hline Liskov \& Wing & $\checkmark$ & $\checkmark$ & $\checkmark$ & $\checkmark$ & N/A & N/A & N/A \\
\hline POOL & $\checkmark$ & $\checkmark$ & $\checkmark$ & & N/A & N/A & N/A \\
\hline
\end{tabular}

Table 1: Differences in subtyping relationships

It is clear from the preceding description that the type models presented can express distributed system concepts to varying degrees. Difficulties arise when applications from different domains (with different type models) must interwork. There are two basic models of interoperability which can be applied to achieve interworking:

- creating a common framework for types including relationship types (in particular subtyping and inheritance relationships), 
- supporting cooperation based on 'known differences', i.e. define one-to-one mappings between type models (which may incur losses and gains in expressiveness).

When presenting a scenario of cooperation between the classes of distributed systems defined above, we apply the 'known differences' approach. This has the disadvantage of incurring an $n(n-1)$ mapping problem, but illustrates the differences in subtyping relationships and shows a possible solution. The 'known differences' approach could be easily mapped to a common framework, provided that the latter is given.

\section{INTERWORKING SCENARIO}

In this section we describe an interworking scenario for the platforms (DCE, CORBA, ANSAware) and models (RM-ODP, Liskov \& Wing's approach) compared earlier. The models are also included, because they forecast future changes in building distributed systems and some of them already have partial implementations. The scenario highlights the differences in type systems and proposes a working solution based on the 'known differences' approach to ensure type safe cooperation between the described systems. For the scenario the following assumptions were made:

- the scenario is restricted to compatibility relationships in heterogeneous distributed systems - other type system differences must be considered, but are not fully presented here,

- the scenario considers only interface extension, component renaming, parameter subtyping, and parameter conversion.

The basis for cooperation is provided by persistent type information in each domain. We call the objects managing this type information "type managers". They are responsible for resolving type differences among heterogeneous systems. For the purpose of this scenario we assume that type managers for the domains are centralised. We focus on information about type system differences only, not on the management of this information.

\subsection{Federation of type managers}

To cope with the differences between type systems of various distributed domains it is essential that the type managers federate. The federation can be limited to subdomains. The federation process includes learning about the differences in type systems and possible losses which may be incurred by translation from one type system to another. In our scenario only differences and losses in inclusion polymorphism are emphasised. We assume that starting points for querying type lattices in type managers are defined prior to resolution of subtyping differences. When the federation process is accomplished, the federated type managers are able to perform inter-domain type matching automatically with the limitations of the matching already known to the domains. A cut-down view of an example federation is shown in Figure 1.

\subsection{The banking scenario}

To create a scenario of interworking between the domains we consider a family of banking interfaces and we examine how previously described type domains are able to interpret relationships among the interfaces. All the approaches to inclusion polymorphism described earlier 


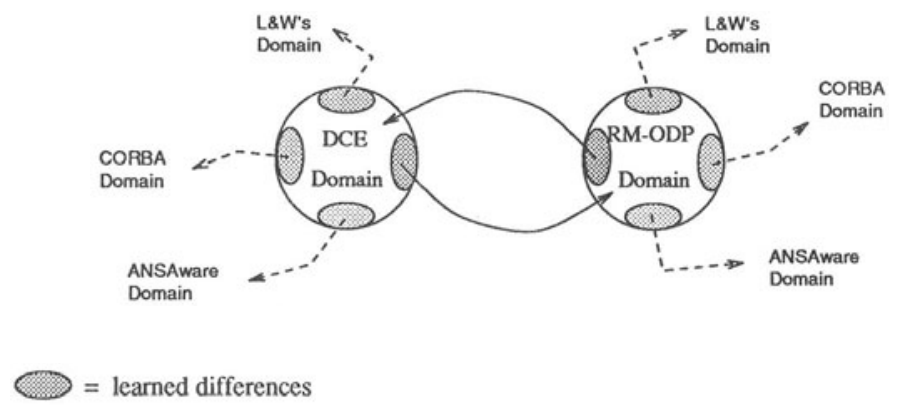

Figure 1: An example federation

are illustrated in this scenario. For each model we analyse the representation of its subtyping relationship and the mapping of the relationships which can be used for interworking. Each type manager must know the subtyping relationships of other federated domains and be able to map its own relationship onto the others. In other words, during federation the relationships between subtyping relationships are established.

The remainder of this section analyses the basis for establishing relationships among subtyping relationships while illustrating it using real life examples of substitutability for banking applications. Semantic subtyping which is present only in Liskov \& Wing's (L\&W) model has not been fully addressed in the scenario because the result of mapping is obvious. No subtype relationship in any of the domains can be accepted as a subtype in the L\&W domain without a loss. The result of a query to other domains can only give a 'hint' about possible subtyping relationship among the returned types, but not the exact answer. Querying the L\&W domain from any other domain is different. Everything allowed by the structural subtyping described below can be accepted if the semantics are added.

We start with a simple banking interface:

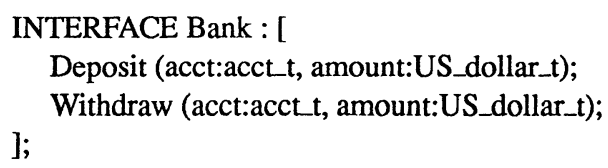

\subsubsection{Interface extension}

Let us assume that there also exists a bank with the following interface:

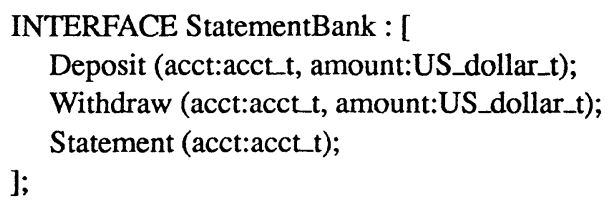

StatementBank is a subtype of Bank because it extends the interface by the addition of an operation. The analysis of the five models leads to the following observations: 
- DCE uses sequential minor version numbers to represent the compatibility relationship ... Bank v1.0, StatementBank v1.1. Interface operation order must be identical.

- ANSAware uses type conformance to specify that StatementBank is a subtype of Bank.

- CORBA uses interface inheritance. StatementBank inherits from Bank.

- RM-ODP uses the definition of subtyping relationship to recognise that StatementBank is a subtype of Bank (by comparing the syntax of the two).

- L\&W uses the definition of the subtyping relationship to check relationships between common operations and defines an extension mapping between Bank and StatementBank. However, these two interfaces are in a subtype relationship if pre- and post-conditions are also in a subtype relationship as defined by the model.

As can be seen, the mapping of an extension-based subtyping relationship between type models is straightforward in all cases and the translation between domains will not incur any loss of information (assuming that the semantic losses for the L\&W domain are not considered).

\subsubsection{Component renaming}

Let us assume, that another bank is available, which provides an equivalent service, but names of the interface, operations and parameters and types differ:

INTERFACE BanqueFrancaise : [

Deposer (compte:compte_t, somme:US_dollar_t); ];

Retirer (compte:compte_t, somme:US_dollar_t);

The analysis of the models leads to the following results:

- DCE cannot legitimately represent the relationship between Bank and BanqueFrancaise. The simple versioning subtyping scheme could be used as long as the types are mapped into basic types, because interactions between clients and servers involves no names, only numbered operations, parameters, and base type references. However this is an exploitation of implementation-specific details of current DCE systems.

- ANSAware uses type conformance to specify the relationship between Bank and BanqueFrancaise, however again this relies on the fact that the ANSAware implementation does not check names during invocation.

- CORBA (OMA) interface subtyping only stipulates that the operation names and parameter types must be equivalent, but that parameter names can change. As the operation names are different, the relationship between types is not recognised.

- RM-ODP will not recognise any relationship between Bank and BanqueFrancaise.

- L\&W uses its subtyping definition which allows renaming. It will recognise the relationship provided that a renaming map is defined, and that any pre- and post-conditions are also in a subtype relationship. 
As the evaluation shows, the mapping between relationships can incur some losses. For example, when a query about types matching Bank will be forwarded from CORBA (or an RM-ODP-like system) to the L\&W domain, the result of the query will have to be filtered in the CORBA type manager to remove all subtypes with renamed operations or parameter types. DCE and ANSAware would be able to cope with renamed parameters in some cases (parameter names), but in others the result would have to be filtered. L\&W's model is able to take the result of the query from any other domain (again semantic differences are ignored).

\subsubsection{Parameter subtyping}

Let us assume, that there exists a banking interface which accepts only small transactions:

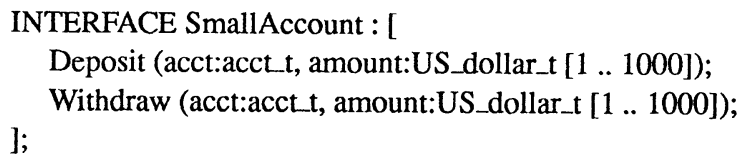

If we analyse the subtyping relationships of our five models, the conclusions are:

- DCE, ANSAware and CORBA have no means to represent subtyping on subranges, and will not be able to determine that Bank is a subtype of SmallAccount,

- RM-ODP and L\&W apply their respective subtyping definitions to recognise the relationship between SmallAccount and Bank.

Translation from RM-ODP or L\&W's model to DCE or ANSAware or CORBA will lose all parameter subtyping information.

\subsubsection{Parameter type conversion}

The last stage of the scenario is not directly related to subtyping relationships. It tries to illustrate that in real heterogeneous distributed systems, a more general meaning for compatibility should be assumed than subtyping provides. Let us take into account the following interface:

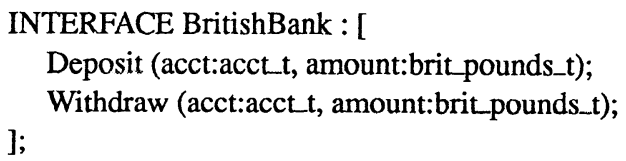

If we assume that brit_pound $t$ can be converted to $U S_{-}$dollar_t $t$ then Bank and BritishBank are compatible (provided that the conversion is made). Some of the compared models may incorrectly believe that Bank and BritishBank are in a subtype relationship (in both directions, i.e. they are equivalent). None of the models has sufficient expressive power to show the constraints of this compatibility (conversion required). Liskov \& Wing's mapping rules associated with relationships are most applicable to this problem, but does not provide the full solution. To provide an 'open' compatibility, a general type relationship mechanism must be introduced which will allow the definition of relationship types and mapping among them including various kinds of equivalence, subtyping and conversion relationships [11]. 


\subsubsection{Summary}

The scenario presents examples of banking interfaces and applies to them various kinds of subtyping on interfaces. It also suggests possible mappings between subtyping relationships when type managers of the various domains federate provided that the type managers can understand relationship types and are able to relate them (e.g. the type manager presented in [4]). Table 2 summarises the possible relationship translations including interface extension, renaming, parameter subtyping, and parameter conversion. The information about these translations has to be introduced to the type managers during federation and used for implementing 'filters' for the results of type queries directed to other domains. The left column in the table shows the model to be translated "from" and the top the translated "to" models. Those marked with a dagger incur some loss of information in the translation as noted in the previous sections.

\begin{tabular}{|c||c|c|c|c|c|}
\hline & DCE & \multicolumn{1}{|c|}{ ANSAware } & \multicolumn{1}{|c|}{ CORBA } & \multicolumn{1}{|c|}{ RM-ODP } & \multicolumn{1}{|c|}{ LW } \\
\hline \hline DCE & $*$ & $\begin{array}{l}\text { extension, } \\
\text { renaming }\end{array}$ & $\begin{array}{l}\text { extension, } \\
\text { renaming }\end{array}$ & extension & $\begin{array}{l}\text { extension, } \\
\text { renaming }\end{array}$ \\
\hline ANSAware & $\begin{array}{l}\text { extension, } \\
\text { renaming }\end{array}$ & $*$ & $\begin{array}{l}\text { extension, } \\
\text { renaming }\end{array}$ & extension & $\begin{array}{l}\text { extension, } \\
\text { renaming }\end{array}$ \\
\hline CORBA & $\begin{array}{l}\text { extension, } \\
\text { renaming }\end{array}$ & $\begin{array}{l}\text { extension, } \\
\text { renaming } \dagger\end{array}$ & $*$ & extension & $\begin{array}{l}\text { extension, } \\
\text { renaming }\end{array}$ \\
\hline RM-ODP & extension & extension & extension & $\begin{array}{l}\text { extension, } \\
\text { parameter } \\
\text { subtyping }\end{array}$ \\
\hline L\&W & $\begin{array}{l}\text { extension, } \\
\text { renaming }\end{array}$ & $\begin{array}{l}\text { extension, } \\
\text { renaming }\end{array}$ & $\begin{array}{l}\text { extension, } \\
\text { renaming } \dagger\end{array}$ & $\begin{array}{l}\text { extension, } \\
\text { parameter } \\
\text { subtyping }\end{array}$ & $*$ \\
\hline
\end{tabular}

Table 2: Possible relationship translations

\section{CONCLUSION}

The paper has examined interoperability features of well known platforms for distributed computing (DCE, ANSAware, CORBA) and some models built for distributed and objectoriented systems (including RM-ODP). The purpose of the examination was to highlight the differences in their type systems which make type safety of interoperability among heterogeneous distributed domains difficult. Requirements for interoperability have been discussed. An examination was carried out showing differences in the definition of data types, action types and relationship types, and in particular types of compatibility relationships. The latter were also illustrated by a cooperation scenario based on one-to-one mappings between various kinds of compatibility relationships. These mappings can be a basis for creating a federation of the heterogeneous domains. Through highlighting the differences, the comparison also facilitates work on a future common interoperability framework to capture the differences among the type systems.

\section{REFERENCES}

[1] P. America. "Designing an Object-Oriented Programming Language with Behavioural Subtyping". In Foundations of Object-Oriented Languages, number 489 in Lecture Notes in Computer Science, pages 60-90, REX School/Workshop, Noordwijkerhout, The Netherlands, 1991. Springer-Verlag. 
[2] APM Ltd, Cambridge UK. ANSAware 4.1 Application Programmer's Manual, Mar. 1992. Document RM.102.00.

[3] J. Auerbach, A. Goldberg, G. Goldszmidt, A. Gopal, M. Kennedy, J. Russell, and S. Yemini. "Concert/C Specification: Definition of a Language for Distributed C Programming". Technical Report RC18994, IBM T. J. Watson Research Center, July 1993.

[4] C. J. Biggs, W. Brookes, and J. Indulska. "Enhancing Interoperability of DCE Applications: a Type Management Approach". In Proceedings of the First International Workshop on Services in Distributed and Network Environments, SDNE'94, 1994.

[5] A. Black, N. Hutchinson, E. Jul, H. Levy, and L. Carter. "Distribution and Abstract Types in Emerald". IEEE Transactions on Software Engineering, SE-13(1):65-76, Jan. 1987.

[6] W. Brookes, A. Berry, A. Bond, J. Indulska, and K. Raymond. "A type model supporting interoperability in open distributed systems". In Proceedings of the First International Conference on Telecommunications Information Networking Architecture, TINA '95, Feb. 1995.

[7] V. Cahill, R. Balter, N. Harris, and X. Rousset de Pina. The COMANDOS Distributed Application Platform. Volume 1. ESPRIT Research Reports (Project 2071). Springer-Verlag, 1993.

[8] L. Cardelli and P. Wegner. "On Understanding Types, Data Abstraction, and Polymorphism". Computing Surveys, 17(4):471-522, Dec. 1985.

[9] R. A. de By and H. J. Steenhagen. "Interfacing Heterogeneous Systems through Functionally specified Transactions". In D. K. Hsiao, E. J. Neuhold, and R. Sacks-Davis, editors, Proceedings of IFIP DS-5 Semantics of Interoperable Database Systems, volume 2, pages 38-45, Lome, Victoria, Australia, Nov. 1992.

[10] S. J. Garland, J. V. Guttag, and J. J. Horning. "An overview of Larch". In Functional Programming, Concurrency, Simulation and Automated Reasoning, number 693 in Lecture Notes in Computer Science, pages 329-348. Springer-Verlag, July 1993.

[11] J. Indulska, M. Bearman, and K. Raymond. "A Type Management System for an ODP Trader". In Proceedings of the IFIP TC6/WG6.1 International Conference on Open Distributed Processing, ICODP'93, (Berlin, Germany, 13-16 September, 1993). IFIP Transactions, Elsevier, North Holland, 1994.

[12] ISO/IEC DIS 10746-3. Draft Recommendation X.903: Basic Reference Model of Open Distributed Processing - Part 3: Prescriptive Model, Apr. 1994. Output of Geneva editing meeting 14-25 February 1994.

[13] B. Liskov and J. M. Wing. "A New Definition of the Subtype Relation". In O. Nierstrasz, editor, Proceedings of the European Conference on Object Oriented Programming, ECOOP '93, number 707 in Lecture Notes in Computer Science, pages 118-141, Kaiserslautern, Germany, July 1993. Springer-Verlag.

[14] B. H. Liskov and J. M. Wing. "A Behavioural Notion of Subtyping". ACM Transactions on Programming Languages and Systems, 1994. (to appear).

[15] F. Manola and S. Heiler. "A "RISC" Object Model for Object System Interoperation: Concepts and Applications”. Technical Report TR-0231-08-93-165, GTE Laboratories Incorporated, Aug. 1993.

[16] E. Najm and J.-B. Stefani. "A formal semantics for the ODP computational model". Computer Networks and ISDN Systems, to appear.

[17] Object Management Group. Object Management Architecture Guide, second edition, Sept. 1992. Revision 2.0, OMG TC Document 92.11.1.

[18] Object Management Group and X/Open. The Common Object Request Broker: Architecture and Specification, 1992.

[19] J. Palsberg and M. I. Schwartzbach. "Three Discussions on Object-Oriented Typing". ACM SIGPLAN OOPS Messenger, 3(2):31-38, 1992.

[20] J. Palsberg and M. I. Schwartzbach. Object-Oriented Type Systems. John Wiley \& Sons, 1994.

[21] W. Rosenberg and D. Kenney. Understanding DCE. Open System Foundation, 1992.

[22] J. Shirley. Guide to Writing DCE Applications. Open System Foundation, 1992. 